\title{
Editorial:
}

\section{Complex systems and networks with their applications}

\author{
Jin-de CAO ${ }^{\dagger 1}$, Yang LIU ${ }^{2}$, Jian-quan LU $^{1}$, Leszek RUTKOWSKI ${ }^{3,4}$ \\ ${ }^{1}$ School of Mathematics, Southeast University, Nanjing 210096, China \\ ${ }^{2}$ College of Mathematics and Computer Science, Zhejiang Normal University, Jinhua 321004, China \\ ${ }^{3}$ Institute of Computational Intelligence, Czestochowa University of Technology, Czestochowa 42-200, Poland \\ ${ }^{4}$ Information Technology Institute, University of Social Sciences, Lódź 90-113, Poland \\ †E-mail: jdcao@seu.edu.cn
}

https://doi.org/10.1631/FITEE.2020000

Complex systems and networks capture most of the features in existing systems and have gained considerable attention over the past several decades. In recent years, research has extended to the fields of networked control systems, cyber-physical systems, logical networks, neural networks, and related topics. This is mainly due to rapid developments in communication, computer, and information technologies, which enable the handling of large-scale data that cannot be easily handled via traditional methods. More importantly, newly invented technologies such as wireless sensor networks, decentralized control, and deep learning techniques need distributed control strategies to ensure their robustness, flexibility, scalability, low cost, and easy configuration and maintenance.

Recent decades have witnessed massive developments and advances in complex systems and networks. Many insightful results and powerful methods have been reported. Complex systems and networks have been broadly applied to both civil and military fields. Typical examples include transportation systems, power grids, health and medical fields, aerospace, and industrial systems. With increasing demands for clean energy, low cost, more security, and better health, the study of complex systems and networks is attracting more attention, from both aca-

\footnotetext{
ॠCorresponding author

(C) Zhejiang University and Springer-Verlag GmbH Germany, part of Springer Nature 2020
}

demic and industrial communities.

In this context, the Chinese Academy of Engineering (CAE) organized a special issue of Frontiers of Information Technology \& Electronic Engineering, focusing on complex systems and networks with their applications. This special issue aims to promote research on complex systems and networks, and to reflect on the most recent advances, with emphasis on both theories and applications. After a rigorous review process, 12 papers by researchers worldwide have been selected for this special issue, including two survey papers and 10 research papers.

Xiao-di LI and his collaborators gave a comprehensive survey of impulsive control strategies, focusing on the field of complex networks. They concentrated on impulsive control and impulsive perturbation, and surveyed several aspects of complex dynamic networks with impulsive effects: synchronizing and desynchronizing impulses, adaptiveimpulsive synchronization, pinning impulsive synchronization, and complex dynamical networks with stochastic and impulsive effects. They also suggested some future research directions. Hai-tao LI and his collaborators carried out a detailed survey on new developments in control design techniques of logical control networks, including the reachable set approach, pinning control design, control Lyapunov function approach, and sampled-data control method.

Jin-de CAO and his collaborators discussed the multiple Mittag-Leffler stability problem for fractional-order quaternion-valued neural networks in 
the presence of impulsiveness, due to the wide applications of neural networks. They showed the existence of equilibrium points using a tool derived from the geometrical properties of activation functions when the Lipschitz condition is assured. With the help of the Lyapunov technique, they also examined the condition of the global Mittag-Leffler stability of multiple equilibrium points for impulsive fractional-order quaternion-valued neural networks. The methods developed were carefully verified by numerical examples.

With the rapid developments in logical networks, Wei-hua GUI and his collaborators proposed an output feedback stabilizer design of a Boolean network (BN) based on the network structure. Unlike traditional pinning control designs, their design is not based on the state transition matrix of BNs, and can efficiently determine the pinning control nodes and reduce computational complexity. Their simulations and experiments using a signal transduction network and a $D$. melanogaster segmentation polarity gene network showed good performance and adaptability of the method.

Logical networks as a typical formalism to model genetic regulatory networks has been a hotspot of interest, and found wide applications in fields such as systems biology, power grids, combustion engines, game theory, encryption, and robotics. Since the development of the algebraic state space approach, it has become easier to analyze the structure and dynamics, and to consider the control problems of logical control networks, including Boolean and multivalued logical networks.

Jian-quan LU and his collaborators reported switching-based stabilization of aperiodic sampleddata Boolean control networks with all subsystems that are unstable. Their method can not only solve the global stability problem of a Boolean control network under aperiodic sampled-data control when all subsystems of the transformed switched network are unstable, but also be adapted for research on the global stability of switched Boolean networks containing all subsystems that are unstable. Compared with research on the global stability of switched Boolean networks with all unstable subsystems, the problem of aperiodic sampled-data with all subsystems that are unstable is more complex.
In recent years, synchronization and consensus phenomena have attracted much attention in attempts to understand how collective behavior emerges in networked systems. Chi HUANG and his collaborators presented a new method on bisimulation-based stabilization of probabilistic Boolean control networks with state feedback control, which can be used to reduce the high computational complexity. A necessary and sufficient condition was put forward based on a powerful matrix manipulation, and the propagation of stabilization with a probability of 1 between bisimilar probabilistic Boolean control networks was considered and demonstrated attainable. Lulu LI and his collaborators investigated the complete synchronization problem of coupled delayed Boolean networks, where the state delays and output delays may not be equal, and the state delay in each Boolean network may be different. One necessary and sufficient condition was proposed for complete synchronization, and a corresponding algorithm was given.

Jin-feng PAN and Min MENG considered optimal one-bit perturbation in Boolean networks based on cascading aggregation, which maximizes the size of basins of attractions of desired attractors and minimizes that of undesired attractors. Based on an aggregation method, a necessary and sufficient condition was given to ensure the invariance of desired attractors after one-bit perturbation. Their experiments on a T-cell receptor network demonstrated the validity, feasibility, and effectiveness of the proposed method.

Cascading decomposition, as an essential and special form of decomposition of Boolean control networks, is a theoretically interesting and practically useful concept. Yi-feng LI and Jian-dong ZHU reported their study of two types of cascading decomposition problems of Boolean control networks using a graph-theoretical method. A new graphic concept called "nested perfect equal vertex partition" was proposed; necessary and sufficient graphic conditions were then obtained. In addition, the logical coordinate transformations were constructively obtained to realize the corresponding cascading decomposition forms. The validity, feasibility, and effectiveness of their proposed method were shown by experimental data.

Because of time-consuming processes involved in DNA transcription and RNA translation, time delay 
is inevitable in the gene regulatory process, and plays a key role in the development of living organisms. Recently, several interesting results have been reported from using delayed logical control networks. In this issue, Ya-ting ZHENG and Jun-e FENG described their investigation into the output tracking problem of delayed logical control networks with state and control constraints under transformation into a set stabilization issue. Based on a deformation of the state transition matrix, several necessary and sufficient conditions were derived, and a design of admissible state feedback controllers was proposed. Their experiments demonstrated the validity, feasibility, and effectiveness of the proposed method.

Recently, there have been rapid developments in neural networks encouraged by their potential applications, such as in medical image edge detection, medical event detection, pattern recognition, and robotics. Yang CAO and his collaborators reported their study of inertial bidirectional associative memory neural networks subject to mixed delays and impulsive effects. With the aid of differential inequality techniques, they provided criteria guaranteeing the existence, uniqueness, and global exponential stability of anti-periodic solutions in the considered system. An example was presented to show the efficiency of the results.

Accessibility of traffic information has enormous potential to automotive powertrain control. Ming-xin KANG and Jin-wu GAO described a gearshift control scheme in which the control design gearshift strategy can be formulated within the framework of the logical system. Towards this goal, vehicle dynamics were modeled by a Markov process. The fuel optimization problem was formulated via a receding horizon optimal control problem in the framework of the logical system. A tool from dynamic programming was used to solve the underlying optimal problem. Simulation results were given to support these designs.

The papers included in this special issue cover a broad spectrum of current research topics on logical networks, complex systems, and neural networks, including stabilization, tracking and decomposition problems of logical networks, analysis and synthesis on neural networks, synchronization problems in complex networks via impulsiveness-based control strategies, and even the optimization of fuel efficiency of vehicles. We hope that this special issue will benefit researchers in these fields and foster research on complex systems and logical networks, neural networks, and related fields.

This special issue would not be possible without the support of many people, including the authors and reviewers. We are indebted to the editorial staff of the journal for their enormous assistance, and to the Editors-in-Chief, Profs. Yun-he PAN and Xi-cheng $\mathrm{LU}$, for this great showcase.

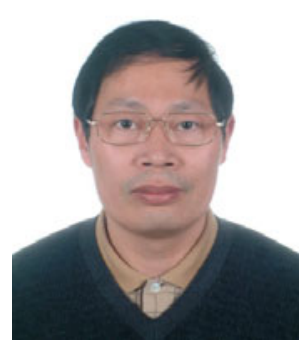

Prof. Jin-de CAO received a BS degree from Anhui Normal University, China, an MS degree from Yunnan University, China, and a $\mathrm{PhD}$ degree from Sichuan University, China, all in mathematics/applied mathematics, in 1986, 1989, and 1998, respectively. He is an Endowed Chair Professor, Dean of the School of Mathematics, Director of the Jiangsu Provincial Key Laboratory of Networked Collective Intelligence of China, and Director of the Research Center for Complex Systems and Network Sciences at Southeast University. Prof. CAO is a recipient of the National Innovation Award of China, Obada Prize, and the Highly Cited Researcher Award in Engineering, Computer Science, and Mathematics by Thomson Reuters/Clarivate Analytics. He has been elected a fellow of IEEE, a member of the Academy of Europe, a member of the European Academy of Sciences and Arts, a fellow of the Pakistan Academy of Sciences, and an Academician of the International Academy for Systems and Cybernetic Sciences. His research interests include complex network and complex system, neurodynamics and optimization, and multi-agent systems.

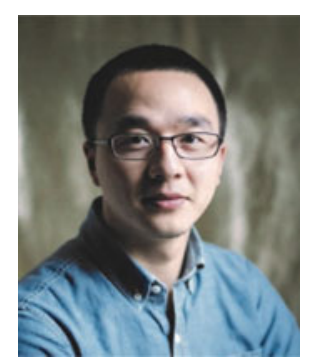

Prof. Yang LIU received a BS degree in mathematics from Zhejiang Normal University, China, in 2003, and a $\mathrm{PhD}$ degree from Tongji University, China, in 2008. He is currently a professor at the College of Mathematics and Computer Science of Zhejiang Normal University. He was the recipient of the Shanghai Outstanding PhD Thesis Award in 2012. He is an Associate Editor of Neur Process Lett. His research interests include hybrid systems and logical systems. 


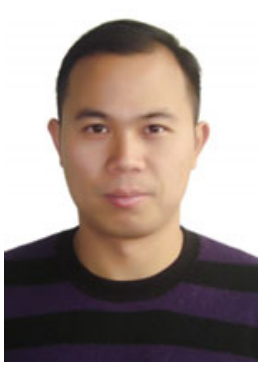

Prof. Jian-quan LU received a BS degree from Zhejiang Normal University, China, an MS degree from Southeast University, China, and a $\mathrm{PhD}$ degree from City University of Hong Kong, Hong Kong, China, all in mathematics/ applied mathematics, in 2003, 2006, and 2009, respectively. From 2010 to 2012, he was an Alexander von Humboldt Research Fellow in PIK, Germany. He is currently a professor at the Department of Mathematics, Southeast University, China. He has published over 70 papers in refereed international journals. He is an associate editor of Neur Process Lett, J Franklin Inst, and Neur Comput Appl, and a guest editor of Math Comput Simul and IET Contr Theory Appl. He was awarded an Alexander von Humboldt Fellowship in 2010. He was recognized by the Program for New Century Excellent Talents in University by the Ministry of Education, China in 2010, by Elsevier as a Most Cited Chinese Researcher in 2014-2016, and by Clarivate Analytics as a Highly Cited Researcher in 2018 and 2019. His current research interests include collective behavior in complex dynamic networks and multi-agent systems, and Boolean control networks.

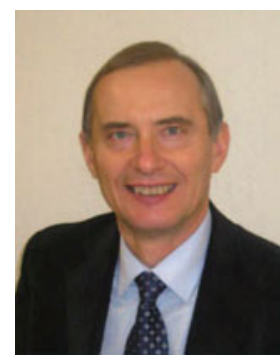

Prof. Leszek RUTKOWSKI received $\mathrm{MS}$ and $\mathrm{PhD}$ degrees from the Wroclaw University of Technology, Wroclaw, Poland, in 1977 and 1980, respectively. He has been with the Czestochowa University of Technology, Czestochowa, Poland, since 1980, where he is currently a professor and Director of the Institute of Computational Intelligence. From 1987 to 1990, he held a visiting position with the School of Electrical and Computer Engineering, Oklahoma State University, Stillwater, OK, USA. He has authored over 200 publications and six books. He is the Founding Chair of the Polish Chapter of the IEEE Computational Intelligence Society, which received the 2008 Outstanding Chapter Award. In 2004, he was elected a member of the Polish Academy of Sciences. He was awarded the IEEE Fellow Membership Grade for contributions to neurocomputing and flexible fuzzy systems in 2004. He was a recipient of the IEEE Trans Neur Netw Outstanding Paper Award in 2005. In 2014 he received a degree honoris causa from the prestigious AGH University of Science and Technology in Krakow, Poland. His current research interests include stream data mining, computational intelligence, and pattern classification. 